\title{
Antituberculosis Drug-Induced Liver Injury: An Ignored Fact, Assessment of Frequency, Patterns, Severity and Risk Factors
}

\author{
Iftikhar Haider Naqvi1 ${ }^{*}$, Khalid Mahmood ${ }^{1}$, Abu Talib ${ }^{1}$, Aamer Mahmood ${ }^{2}$ \\ ${ }^{1}$ Department of Medicine, Dow University of Health Sciences, Karachi, Pakistan \\ ${ }^{2}$ Agha Khan University Hospital, Karachi, Pakistan \\ Email: "drihnaqvi@gmail.com
}

Received 4 November 2015; accepted 13 December 2015; published 16 December 2015

Copyright (C) 2015 by authors and Scientific Research Publishing Inc.

This work is licensed under the Creative Commons Attribution International License (CC BY). http://creativecommons.org/licenses/by/4.0/

(c) (i) Open Access

\begin{abstract}
Background/Aims: Antituberculosis drug-induced liver injury (TB DILI) is a frequent medical problem in Pakistan. Critical understanding of various aspects of TB DILI is not only important to manage liver injury but may also prevent unnecessary discontinuation of antituberculosis treatment. The study is aimed to determine the frequency, types, severity and patterns of TB DILI. Study further evaluates various risk factors of TB DILI. Materials and Methods: This is a prospective cohort study of two seventy-eight patients with the diagnosis of tuberculosis, where patients were followed during tuberculosis treatment. TB DILI was defined in accordance to international DILI expert working group. Results: Out of two seventy eight-patients, ninety-five $(34.14 \%)$ had TB DILI. The most common pattern of TB DILI was hepatocellular $(63.15 \%)$ followed by mixed (23.15\%) and Cholestatic (13.68\%). Most of the patients had mild DILI (43.15\%) followed by moderate $(30.52 \%)$, severe $(20.01 \%)$ and very severe $(5.26 \%)$. Age $>35$ years, concomitant hepatotoxic drugs, extrapulmonary TB and malnutrition are important risk factors for TB DILI. Conclusion: All patterns of TB DILI with varying severity were present. Age > 35 years, malnutrition, extrapulmonary TB and concomitant use of hepatotoxic drugs were risk factors for TB DILI.
\end{abstract}

\section{Keywords}

Antituberculosis Drug-Induced Liver Injury, Antituberculosis Treatment, Subjective Global Assessment, Liver Function Test

\section{Introduction}

Tuberculosis (TB) continues to be a major health problem, with 9.4 million incident cases and 1.7 million deaths

${ }^{*}$ Corresponding author.

How to cite this paper: Naqvi, I.H., Mahmood, K., Talib, A. and Mahmood, A. (2015) Antituberculosis Drug-Induced Liver Injury: An Ignored Fact, Assessment of Frequency, Patterns, Severity and Risk Factors. Open Journal of Gastroenterology, 5, 173-184. http://dx.doi.org/10.4236/ojgas.2015.512027 
globally in 2009 [1]. Tuberculosis perseveres as an important infectious disease across most of the underdeveloped world. It explains a noteworthy socioeconomic strain. The prevalence of tuberculosis in Pakistan is 350 (158 - 618)/100,000 with a mortality rate of 33 (15 - 60)/100,000 [2]. Despite the declining trend in prevalence and incidence of tuberculosis in the west, the HIV/AIDS rise has set hurdles for its control in developing countries [3].

TB DILI (Antituberculosis drug-induced liver injury) is defined as the liver injury as a result of antituberculous drugs in accordance to American Thoracic society and international DILI Expert Working Group. TB DILI (Antituberculosis drug-induced liver injury) is multifactorial including metabolic idiosyncrasy to HLA susceptibility and generation of various free radical species. Metabolic idiosyncrasy claims to be the basis of Isoniazid (INH) toxicity where a CYP-generated toxic metabolite of INH induces oxidative stress causing mitochondrial or other key organelles injury. This concept could explain the enhanced risk of severe liver injury with concomitant intake of rifampicin and pyrazinamide [4]. HLA susceptibility with certain HLA haplotypes where HLADQB1 0201 antigen is present where HLA-DQA1*0102 is absent, has been linked with INH induced liver injury [5].

Rifampicin may itself be hepatotoxic, as observed in cases with positive rechallenge and in situations where it is used alone (as to relieve pruritus). The meta-analysis showed that regimens combining isoniazid and rifampicin were associated with a higher rate of hepatotoxicity (2.5\%) than regimens without this combination (1.1\%) [6]. Pyrazinamide exhibits a wide range hepatotoxicity which includes idiosyncratic dose-dependent, sporting eosinophilia and granulomatous hepatitis. Pyrazinamide modifies nicotinamide acetyl dehydrogenase levels consequently generating free radical species.

Regardless of great advantages with a combination of several antitubercular drugs having synergistic effects, effectivity against different populations of mycobacterium, prevention of drug resistance and the risk of hepatotoxicity of individual drugs is increased. Even though majority of the patients endure the combination of drugs, a few develop adverse effects where anti-tuberculosis treatment induced hepatoxicity is the most significant adversity [7] [8] accounting for more than $7.0 \%$ of all undesired effects [9] [10]. The incidence of TB DILI varied from $2.0 \%$ to $28.0 \%$ which is based on different populations and definitions [7].

TB DILI includes liver damage ranging from a minimal elevation of ALT or AST without symptoms to acute hepatic failure often fatal or even requiring liver transplantation.

Asymptomatic derangement of liver enzymes is found in $20 \%$ of patients (as a consequence of adaptation) [11] [12]. The less favorable outlook has been reported in patients who develop jaundice, acute liver failure, ascites and encephalopathy [13]. Moreover, TB DILI reduces efficacy of anti-TB treatment which could cause noncompliance, treatment failure and eventual recurrence or drug-resistance [14]. These consequences could momentously hamper effective epidemic TB control.

Various risk factors for TB DILI have been evidenced as old age, female gender, high alcohol ingestion, coadministration of enzyme inducers \& other hepatotoxic drugs, Asian ethnicity and advanced form of the disease. Prior viral related (HBV \& HCV) chronic liver disease, human immunodeficiency virus (HIV) infection and malnutrition are also established risk factors for TB DILI [7] [15] [16].

Pakistan is an endemic country for TB where various forms of TB are responsible for a mortality rate of 33 (15 - 60)/100,000 [2]. TB DILI leads to treatment failure that may further exacerbate drug resistance causing eventual collapse of TB eradication programme. Critical understanding of clinical features of TB DILI, such as the time of onset, degree of severity, early symptomatology and potential outcomes are of crucial importance in identification of the adverse events with prompt intervention. A primary health care physician should know when to stop anti-tuberculosis treatment (ATT) in case of DILI. Physicians should have comprehensive knowledge of reintroducing strategy as holding ATT even after DILI resolution may lead to rise in TB resistance and can further hamper TB eradication program in Pakistan. The study is designed to:

- Determine the frequency, types, severity and patterns of TB DILI.

- Evaluate various risk factors for TB DILI.

\section{Materials and Methods}

This is a prospective cohort study of 278 patients with a diagnosis of Tuberculosis. Local institutional ethics committee approval was sought before commencing the study. The study was conducted at Civil Hospital Ka- 
rachi, Pakistan. All confirmed cases of Tuberculosis of age $\geq 18$ years who had given informed consent were included over a period of eight months after commencement of study. Patient with prior history, evidence of liver injury, suspected multi-drug resistant (MDR) and extensively drug resistant (XDR) TB were excluded.

\subsection{Tuberculosis Infection}

TB was diagnosed by WHO criteria including; a positive culture for Mycobacterium tuberculosis or negative culture with clinical and radiological features, response to anti-tuberculosis treatment or over histological findings. In accordance to Pakistan Society of Chest, treatment recommendations for TB [17] a regimen based on isoniazid, rifampicin, pyrazinamide and ethambutol was given for bactericidal or initial phase. Sterilization or continuation phase of treatment was completed with isoniazid and rifampicin.

\subsection{Drug Induced Liver Injury (DILI)}

Drug induced liver injury was defined by American Thoracic society which is further endorsed by international DILI Expert Working Group [18]. For labeling DILI any one of the following criteria should be met:

- Rise in alanine aminotransferase (ALT) more than or equal to fivefold Increase from upper limit of normal (ULN)

- Twofold or more than twofold rise in alkaline phosphatase (ALP) along with associated more than or equal to twofold elevations in concentrations of 5 nucleotidase or $\gamma$-glutamyl transpeptidase above the ULN without known bone pathology.

- More than or equal to three threefold rises in ALT concentration and concurrent elevation of bilirubin concentration surpassing 2 times of the ULN.

\subsection{Pattern of DILI}

A pattern of drug induced liver injury (DILI) was established by applying criteria proposed by International DILI Expert Working Group [18]. Pattern of DILI is defined by using R-value where R = (ALT/ULN)/(ALP/ULN) and alkaline phosphatase (ALP) from the same serum sample.

- $\mathrm{ALT}$ activity = patient's ALT/upper limit of normal (ULN); ALP activity = patient's ALP/ULN; R = ALT activity/ALP activity.

- Hepatocellular pattern of DILI $=\mathrm{R} \geq 5$.

- $\quad$ Mixed pattern of DILI $=\mathrm{R}>2$ and $<5$.

- Cholestatic pattern of DILI $=\mathrm{R} \leq 2$.

\subsection{DILI Severity Index}

The severity of DILI was graded in accordance with International DILI Expert Working Group [18], where severity of DILI graded as follow:

Mild: Elevated alanine aminotransferase or alkaline phosphatase concentration reaching criteria for DILI* but bilirubin concentration $<2 \times$ upper limit of normal (ULN).

Moderate: Elevated alanine aminotransferase or alkaline phosphatase concentration reaching criteria for DILI $^{*}$ and bilirubin concentration $\geq 2 \times U L N$, or symptomatic hepatitis.

Severe: Elevated alanine aminotransferase or alkaline phosphatase concentration reaching criteria for DILI* and bilirubin concentration $\geq 2 \times \mathrm{ULN}$, and at least one of the following:

- International normalized ratio $\geq 1.5$.

- Ascites and/or encephalopathy, disease duration <26 weeks, and absence of underlying cirrhosis.

- Other organ failure considered to be caused by DILI.

Fatal: Death or liver transplantation for drug induced liver injury.

" (proposed by International DILI Expert Working Group).

\subsection{Other Laboratory Test}

All baseline investigations were done before initiation of anti-TB drugs including complete blood counts, renal function tests (serum creatinine and blood urea) and both viral markers i.e.; hepatitis B surface antigen (HBsAg) 
and anti-hepatitis C antibody (anti-HCV). Samples from HBsAg + Ve, anti-HB core antibody (anti-HBc) + Ve, and/or anti-HCV antibody-positive patients were tested for HBV DNA and HCV RNA, respectively. Liver function tests including alanine aminotransferase (ALT), aspartate aminotransferase (AST), alkaline phosphatase (ALP) and bilirubin were done before initiating anti-TB drugs and subsequently at 1, 2, 4, and 6 weeks later. Patients were also pursued clinically during anti-tuberculosis therapy.

\subsection{Exclusion of Other Causes of Liver Disease}

Before attributing DILI to anti-TB drugs, other etiologies of liver diseases were excluded by: IgM anti-hepatitis A virus antibody, HBsAg, IgM anti-HBc in addition to the HBV DNA if the HBsAg and/or HBcAb are positive, anti-HCV antibody and HCV RNA if the anti-HCV antibody is positive. Autoimmune screen (anti-nuclear and anti-smooth muscle antibodies), IgM and IgG against CMV and abdominal ultrasounds were done to assess for liver abscesses, focal lesions or any biliary obstruction if so.

\subsection{Monitoring of Adverse Drug Events}

For monitoring, patients were advised to fill in the specially designed proforma to record patients' self-reported sign/symptoms and drug usages. The listed experiences were discomforts including nausea, vertigo, headache, diarrhoea, arthralgia, and paresthesia, visual and auditory abnormal feelings. Symptoms suggestive of hepatitis (such as nausea, anorexia, vomiting, icterus, etc.) were also documented.

\subsection{ATT Reintroducing Strategy}

Treatment with the hepatotoxic drugs (isoniazid, rifampicin, and pyrazinamide) was immediately stopped in patients with ATT DILI (based on DILI criteria). These patients were managed with a modified anti-TB drug regimen comprising of ethambutol, streptomycin, and a fluoroquinolone. Patients were pursued weekly till clinical and biochemical parameters of acute liver injury stabled. ATT was reintroduced according to American Thoracic Society guidelines [7] where Rifampicin was started with maximum dose on day first followed by INH on day 8 adding pyrazinamide on day 15. The maximum doses were calculated according to body weight.

\subsection{Assessment of Risk Factors}

Various factors associated with TB DILI as age, gender, diabetes with fatty liver, concomitant use of hepatotoxic drugs and nutritional status or degree of malnutrition were evaluated.

\subsection{Malnutrition}

Among various risk factors for DILI in patients on ATT, malnutrition was determined by Subjective Global Assessment (SGA) scheme categorizing patients into stage A, B and C [19].

\subsection{Statistical Analysis}

Data are analyzed through Statistical Package for Social Sciences (SPSS, version 16.0; SPSS Inc., Chicago, IL) for Windows. Data are expressed as mean \pm SD and frequencies as appropriate. Data were compared through Student's $t$-test and $\chi^{2}$ analysis for continuous variables and proportions, respectively. Risk factors for TB DILI were evaluated by multivariate logistic regression analyses. Statistical significance was considered at $P<0.05$.

\section{Results}

Out of 278 patients with a diagnosis of TB, 95 (34.14\%) had TB DILI. Most of the patients were male 192 (69.0\%). The most common type of TB was pulmonary 59.39\% followed by extrapulmonary forms like intestinal, peritoneal, lymph nodes, etc. The demographic detail of patients with comorbids is given in Table 1 .

\subsection{Clinical Features}

Most common symptoms were nausea, vomiting, and jaundice. The overall clinical features of patients with TB DILI are highlighted in Table 2. 
Table 1. Demographic profile, types and comorbids of patients on ATT.

\begin{tabular}{ccc}
\hline Variables & $\begin{array}{c}\text { N = Number of patients } \\
\text { Total }=278\end{array}$ & Frequency \% \\
\hline Gender & 192 & $69.0 \%$ \\
Male: Female: & 86 & $30.93 \%$ \\
Age & & $64.38 \%$ \\
$>35$ years & 17 & 35.61 \\
$<3$ years & 99 & \\
Type of tuberculosis & & $55.39 \%$ \\
Pulmonary: & 154 & $13.30 \%$ \\
Intestinal: & 37 & $10.43 \%$ \\
Peritoneal: & 29 & $9.71 \%$ \\
Lymph nodes: & 27 & $4.31 \%$ \\
TB meningitis & 12 & $2.87 \%$ \\
Pott's disease: & 08 & $3.23 \%$ \\
Pericardial: & 09 & \\
Comorbids & & \\
DM & & $(30.93 \%)$ \\
Concomitant hepatotoxic drugs & 86 & $(19.06 \%)$ \\
Extra pulmonary TB & 53 & $(43.88 \%)$ \\
Degree of Malnutrition & 122 & $(25.89 \%)$ \\
SGA-A & 72 & $(40.04 \%)$ \\
SGA-B & 128 & $(28.05 \%)$ \\
SGA-C & 78 & \\
\hline
\end{tabular}

SGS, Subjective global assessment. DM, Diabetes Mellitus.

Table 2. Clinical features among patients with TB DILI.

\begin{tabular}{ccc}
\hline Variables & Number (n) & Frequency \% \\
\hline Symptomatology & & $\mathbf{4 7 . 3 6 \%}$ \\
Nausea & 45 & $\mathbf{4 1 . 1 1 \%}$ \\
Vomiting & 39 & $\mathbf{2 6 . 3 4 \%}$ \\
Anorexia & 25 & $\mathbf{2 2 . 2 1 \%}$ \\
Dizziness & 21 & $\mathbf{1 9 . 1 3 \%}$ \\
Abdominal pain & 18 & $\mathbf{4 5 . 0 2 \%}$ \\
Jaundice & 43 & $3.15 \%$ \\
\hline Encephalopathy & 03 & \\
\hline
\end{tabular}

\subsection{Pattern of DILI}

All three patterns of TB DILI including hepatocellular, mixed and Cholestatic were found. Sixty patients out of 95 (63.15\%) had Hepatocellular pattern while 22 patients (23.15\%) had mixed pattern. A cholestatic pattern was present in only 13 (13.68\%). Patterns of TB DILI are shown in Table 3.

\subsection{Severity of DILI}

Patients from mild to very severe TB DILI were found in this study. 41 (43.15\%) out of 95 patients had mild DILI, 29 (30.52\%) patients had moderate DILI while 20 (20.01\%), had severe and 05 (5.26\%) had very severe DILI. The baseline and serial monitoring of LFTS are revealed in Table 4. The frequency and mean values of transaminases with respect to degree of DILI have been shown in Table 5.

\subsection{DILI after Reintroduction of ATT}

On reintroduction of ATT, 19 patients (20\%) had mild DILI with INH, 13 (13.68\%) patients had mild DILI with 
Table 3. Patterns of DILI in patients on ATT.

\begin{tabular}{cccc}
\hline Patterns of DILI & Number of patients (n) Total = 95 & Frequency (\%) & $\begin{array}{c}\text { R value = ALT activity/ALP } \\
\text { activity mean with SD }\end{array}$ \\
\hline Hepatocellular & 60 & $63.15 \%$ & $5.45 \pm 0.33$ \\
Mixed & 22 & $23.15 \%$ & $3.78 \pm 0.91$ \\
Cholestatic & 13 & $13.68 \%$ & $1.34 \pm 0.74$ \\
\hline
\end{tabular}

DILI, Drug induced liver injury. ALT, Alanine transferase. ALP, Alkaline phosphate.

Table 4. Serial LFT's monitoring in patients on ATT at baseline, $2^{\text {nd }}, 4$ th and $6^{\text {th }}$ week.

\begin{tabular}{cccccc}
\hline Serial LFT & $\begin{array}{c}\text { No of patients } \\
\text { Frequency \% Total }=\mathbf{2 7 8}\end{array}$ & $\begin{array}{c}\text { ALT level U/L } \\
\text { (Mean, SD) }\end{array}$ & $\begin{array}{c}\text { Serum bilirubin } \\
(\mathbf{m g} / \mathbf{d l})\end{array}$ & ALP (U/L) & INR \\
\hline Baseline & 278 & $22.94 \pm 4.00$ & $0.62 \pm 0.08$ & $89.71 \pm 26.54$ & $1.156 \pm 0.33$ \\
$\begin{array}{c}\text { At } \mathbf{2}^{\text {nd }} \text { week } \\
\text { LFT normal }\end{array}$ & $183(65.82 \%)$ & $28.35 \pm 0.38$ & $0.95 \pm 0.07$ & $105.92 \pm 6.65$ & $1.18 \pm 0.007$ \\
LFT deranged & $95(34.17 \%)$ & $54.95 \pm 9.85$ & $1.23 \pm 0.02$ & $180.21 \pm 13.50$ & $1.20 \pm 0.02$ \\
At $\mathbf{4}^{\text {th }}$ week & & & & & $1.44 \pm 0.03$ \\
LFT deranged & $95(34.17 \%)$ & $81.18 \pm 2.56$ & $1.38 \pm 0.05$ & $206.03 \pm 9.51$ & \\
At $\mathbf{6}^{\text {th }}$ week & & & & & $2.81 \pm 0.83$ \\
LFT deranged & $95(34.17 \%)$ & $311.85 \pm 44.13$ & $4.52 \pm 1.439$ & $274.63 \pm 74.60$ \\
\hline
\end{tabular}

LFT, Liver function test. ALT, Alanine aminotrasferase. ALP, Alkaline phosphate. INR, International normalized ratio. *DILI severity criterion was applied.

Table 5. Severity of TB DILI in accordance with DILI expert working group.

\begin{tabular}{ccccc}
\hline Severity of TB DILI & $\begin{array}{c}\text { No of patients } \\
\text { Frequency \% Total }=\mathbf{9 5}\end{array}$ & $\begin{array}{c}\text { ALT level U/L } \\
\text { (Mean, SD) }\end{array}$ & Serum bilirubin (mg/dL) & INR \\
\hline Mild: & $41(43.15 \%)$ & $241.58 \pm 7.21$ & $2.39 \pm 0.31$ & $1.35 \pm 0.29$ \\
Moderate: & $29(30.52 \%)$ & $293.75 \pm 8.31$ & $4.27 \pm 0.24$ & $1.48 \pm .27$ \\
Severe: & $20(20.01 \%)$ & $347.65 \pm 9.5$ & $5.99 \pm 0.34$ & $3.67 \pm 0.14$ \\
Very severe: & $05(5.26 \%)$ & $390.2 \pm 6.37$ & $7.12 \pm 0.29$ & $4.92 \pm 0.245$ \\
\hline
\end{tabular}

ALT, Alanine aminotrasferase. International normalized ratio. DILI, Drug induced liver injury.

rifampicin and only 5 (5.26\%) patients had DILI over reintroduction of Pyrazinamide.

\subsection{Assessment of Risk Factors: Age}

Out of 278 tuberculosis patients, 179 (64.38\%) patients were above 35 years of age. Among patients with this age group 69/179 (38.54\%) had DILI with the calculated odd ratio of (1.76, Confidence Interval; CI 0.94 - 3.13), relative risk of (1.46, CI, 95\% $0.99-2.22)$ and p-value of $0.004^{*}$ as shown in Table 6 .

\subsection{Gender}

Male gender was assessed as a risk factor for DILI. Among 278 tuberculosis patients, 192 (69\%) were male and 114 (31\%) were female. Out of 192 male patients 60/192 (31.25\%) had DILI with calculated odd ratio of 0.66, (CI 95\%, 0.37 - 1.16), relative risk of 0.76, (CI 0.54 - 1.10) and p-value of 0.13 as shown in Table 6.

\subsection{Diabetes}

Out of 278 tuberculosis patients, 86 (30.93\%) had DM. Among patients with DM 50/86 (58.13\%) had DILI with calculated odd ratio of 4.53 (CI 95\% 2.54 - 8.11), relative risk of 2.48 (CI 951.78 - 3.21) and p-value of 0.000 as shown in Table 6. 
Table 6. Risk factors for TB DILI BY univariate analysis.

\begin{tabular}{|c|c|c|c|c|}
\hline Variables & TB DILI & Odd Ratio & Relative Risk & \multirow{2}{*}{ p Value } \\
\hline $\mathrm{N}$ & N, Rate \% & CI (95\%) & CI (95\%) & \\
\hline \multicolumn{5}{|l|}{ Gender } \\
\hline Male $=192$ & $60,31.25$ & $0.66(0.37-1.16)$ & 0.76 & 0.13 \\
\hline Female $=86$ & $35,40.69$ & & $0.54-1.10$ & \\
\hline \multicolumn{5}{|l|}{ Age } \\
\hline$>35$ years $(179)$ & 69, 38.54 & $1.76(0.94-3.13)$ & 1.46 & $0.004^{*}$ \\
\hline$<35$ years $(99)$ & $26,26.26$ & & $0.99-2.22$ & \\
\hline \multicolumn{5}{|l|}{ DM } \\
\hline With DM (86) & $50,58.13$ & $4.53(2.54-8.11)$ & 2.48 & $0.000^{*}$ \\
\hline Without DM (192) & $45,23.43$ & & $1.78-3.21$ & \\
\hline \multicolumn{5}{|c|}{ Concomitant Hepatotoxic drugs } \\
\hline Yes (53) & $35,66.03$ & $5.34(2.69-10.68)$ & 2.47 & $0.000^{*}$ \\
\hline No (225) & $60,26.66$ & & $1.79-3.21$ & \\
\hline \multicolumn{5}{|l|}{ Extra Pulmonary TB } \\
\hline Yes (122) & $65,43.88$ & $2.216(1.32-3.71)$ & 1.56 & $0.001^{*}$ \\
\hline No (156) & 53, 33.95 & & $1.17-2.08$ & \\
\hline \multicolumn{5}{|l|}{ Malnutrition SGA-A } \\
\hline Yes (72) & 18,25 & $0.58(.29-1.61)$ & 0.66 & 0.06 \\
\hline No (206) & $77,37.37$ & & $0.40-1.04$ & \\
\hline \multicolumn{5}{|l|}{ SGA-B } \\
\hline Yes (128) & 26, 20.31 & $0.29(0.19-0.65)$ & 0.44 & $0.001^{*}$ \\
\hline No (150) & 69,46 & & $0.29-0.65$ & \\
\hline \multicolumn{5}{|l|}{ SGA-C } \\
\hline Yes (78) & $51,65.38$ & $6.69(3.63-14.41)$ & 2.97 & $0.001^{*}$ \\
\hline N0 (200) & 44,22 & & $2.16-3.96$ & \\
\hline
\end{tabular}

SGA, Subjective global assessment. DM, Diabetes Mellitus.

\subsection{Concomitant Hepatotoxic Drugs}

Out of 278 tuberculosis patients, 53 (19.06\%) were on other hepatotoxic drugs. Among patients on hepatotoxic drugs 35/53 (66.03\%) developed DILI with calculated odd ratio of 5.34 (CI 95\% 2.69 - 10.68) relative risk of 2.47 (CI 95\%, 1.79 - 3.21) and p-value of 0.000 as shown in Table 6.

\subsection{Extrapulmonary TB}

Out of 278 tuberculosis patients, 122 (43.88\%) had other than pulmonary TB. Among patients with extrapulmonary TB 122/65 (53.27 \%) had DILI with calculated odd ratio of 2.216 (CI 95\%, 1.32 - 3.71), relative risk of 1.56 (CI 95\%, 1.17 - 2.08) and p-value of 0.00 as shown in Table 6.

\subsection{Degree of Malnutrition}

Various degrees of malnutrition according to SGA (Subjective global assessment) were assessed as risk factors 
for DILI in tuberculosis patients. Out of 278 tuberculosis patients, 72 (25.89\%) were categorized in SGA A. Among patients in SGA A 18/72 (25\%) developed DILI with calculated odd ratio of 0.58 (95\% CI .29 - 1.61) relative risk of 0.66 (95\% CI 0.40 - 1.04) and p-value of 0.06 .128 patients with tuberculosis were nutritionally placed in SGA B category. Among patients in SGA B 26/128 (20.31\%) developed DILI with calculated odd ratio of 0.29 (95\% CI 0.19 - 0.65) relative risk 0.44, (95\% CI, $0.29-0.65)$ and p-value of 0.001 . Out of 278 patients, 78 (28.05\%) were categorized into SGA C. Among severely malnourished SGA-C patients 51/7 (65.38\%) had DILI with calculated odd ratio of 6.69, (95\% CI, 3.63--14.41) relative risk 2.97, (95\% CI, 2.16 - 3.96) and p-value of 0.001 as shown in Table 6.

\subsection{Independent Risk Factors}

Among all the risk factors for TB DILI, the use of a concomitant hepatotoxic drug and severe malnutrition SGA B and SGA C were found to be independent risk factors for TB DILI when assessed by multivariate analysis. The odd ratios with p-values are given in Table 7.

\section{Discussion}

The incidence of hepatotoxicity is more common with multidrug regimens for TB compared to INH monotherapy in anti-TB prophylaxis. In this study TB DILI was manifested in $34.17 \%$ which is greater than earlier studies where the frequency was $8 \%$ - 19.8\% [20] [21]. A recent Iranian study has shown similar frequency (31.37\%) of TB DILI [22]. Low frequency of TB DILI (5\%) was reported in a meta-analysis from the west [23] Pulmonary TB was the commonest form of TB in this study whereas an earlier study [24] had lesser frequency of pulmonary TB.

Nausea, anorexia, and vomiting are the main clinical presentation of TB DILI in this study which is congruent to the former studies [25] [26]. All three types of DILI were observed here including asymptomatic patients with raised aminotransferase, symptomatic with raised aminotransferase and patients with acute liver failure/encephalopathy. An earlier study showed various types of TB DILI with acute uncomplicated hepatitis (72.7\%), fulminant hepatic failure (9.1\%) and hepatic encephalopathy (18.2\%) [26]. Kumar et al. reported low frequency (5.7\%) of ATT induced acute liver failure with high mortality [13].

All three major patterns of DILI (hepatocellular, Cholestatic, mixed) were present in this study. Most prevalent (63.15\%) DILI pattern was hepatocellular followed by 23.15\% mixed and 13.68\% Cholestatic type. ATT DILI severity was noted from mild to severe in this study. Majority of patients having TB DILI 41 (43.15\%) had mild DILI, 29 (30.52\%) had moderate DILI while 20 (20.01\%) and 05 (5.26\%) patients had severe and very severe DILI respectively. Khalili et al. [22] have shown moderate (18.7\%), severe (7.8\%) and very severe (4.9\%) hepatotoxicity with ATT. Maukof et al. [24] have shown the severity of TB DILI by using modestly different cut off values for transaminases. In above study mild, moderate and severe DILI were present in 66\%, 26.67\% and $13.33 \%$ patients respectively. The difference in DILI severity from above study to ours was owing to different cut off values for transaminases used in those two studies. A large Chinese study also showed similarly severe TB DILI [25] Locally published study on TB DILI showed severity according to elevated level of trans-

Table 7. Risk factors for TB DILI BY multivariate analysis.

\begin{tabular}{|c|c|c|c|c|}
\hline Variables & TB DILI N Rate, \% & OD Ratio & Confidence interval (CI) 95\% & p Value \\
\hline \multicolumn{5}{|l|}{ Malnutrition } \\
\hline \multicolumn{5}{|l|}{ SGA B } \\
\hline Yes (128) & $2620.31 \%$ & & & \\
\hline No (150) & $6946 \%$ & 0.24 & $0.17-0.55$ & 0.031 \\
\hline \multicolumn{5}{|l|}{ SGA C } \\
\hline Yes (78) & $5165.38 \%$ & & & \\
\hline No (200) & $4422 \%$ & 5.43 & $2.51-9.37$ & 0.05 \\
\hline \multicolumn{5}{|c|}{ Concomitant Hepatotoxic drugs } \\
\hline Yes (53) & 35 66.03\% & & & \\
\hline No (225) & $6026.66 \%$ & 4.21 & $2.13-9.68$ & 0.09 \\
\hline
\end{tabular}

SGA, Subjective global assessment. TB DILI, Antituberculosis drug-induced liver injury. 
aminases (ALT/AST > 2) 22.3\%, (ALT/AST > 3) 14.44\% and (ALT/AST > 5) 2.22\% although hepatotoxicity or DILI grading was not in accordance to the International DILI Expert Working Group which includes bilirubin in grading system [27].

DILI was also documented after reintroduction of ATT in this study. $20 \%$ patients had mild DILI with INH whereas rifampicin and PZA produced DILI in $13.68 \%$ and $5.26 \%$ on reintroduction. Sharma et al. analyzed rechallenge of 3 antituberculosis regimens in patients with TB DILI [28]. In this study reintroducing strategy of ATT were compared in three management groups where group I patients were reintroduced with highest doses of isoniazid, rifampicin, pyrazinamide simultaneously while group II patients were given therapy according to ATS guidelines (RIF trailed by isoniazid for 7 days, followed by pyrazinamide for 7 days, all in the maximum dosages). Patients in group III were reintroduced ATT as British Thoracic Society (BTS) guidelines where isoniazid, Rifampicin, and pyrazinamide were given sequentially by achieving the highest dose of each agent. Sharma et al. accomplished that the re-emergence of DILI was comparable amid the three treatment groups, ( $\mathrm{p}=$ 0.69) [28]. The frequency of DILI over the reintroduction strategy by American thoracic society as used in this study was $3.42 \%$.

In contrast, to study by Sharma et al., the Tahaoglu et al. in their study [29] compare the reintroduction regimens of ATT with maximum doses of antituberculosis agents like pyrazinamide and slow reintroduction of ATT without Pyrazinamide. The above study concluded that reintroduction of ATT with maximum doses were more hepatotoxic [29]. The dissimilarity between the above mentioned studies could be owing to a lesser sample size, the lesser treatment restraining limits and possible adaptation [30]. Khalili et al. [22] has shown the overall hepatotoxicity of RIF, INH and PZA were $1 \%, 3 \%$ and $9 \%$ respectively.

Advance stage of TB, underlying acute or chronic liver disease, various degrees of malnutrition and indiscriminate use of drugs are considered as major factors for higher prevalence of TB DILI in developing countries. Conversely, it is imprecise whether they are independent risk factors for hepatotoxicity.

Age $>35$ year was proven as a risk factor for TB DILI in this study. Latest studies are harmonious to this study where patients over 35 years have 4 fold increased possibility of developing TB DILI [31]. A study reported that the rate of TB DILI encompasses $2 \%$ to $8 \%$ on aging, with an average of $5 \%$ [32]. Other studies accounted that DILI ranges from $22 \%$ to $33 \%$ in patients over 35 years compared with a range from $8 \%$ to $17 \%$ in those younger [4] [33]. Mahmood et al. [20] expressed that older age group was affected more with TB DILI than the younger ones (25.8\% and $14.4 \%$, respectively). In contrast Steele et al. [34] observed liver injury (hepatitis) in $1 \%-6.9 \%$ of children contrasted to $1.6 \%-2.5 \%$ of adult's instituted isoniazid and rifampicin combination; involving all age groups at threat of emerging DILI. Shakya et al. reported that the rate of TB DILI is more in younger sufferers [35]. This could be elucidated by the fact that the majority of patients in their study were young. A prior Iranian [22] study disapproved age $>35$ year to be a risk for developing TB DILI.

The feminine gender is conventionally considered more liable to develop TB DILI. Numerous studies reported increased risk of hepatotoxicity, neither treatment limiting nor the statistically significant [36] [37]. Papastavros et al. showed a 4-fold rise in the risk of treatment limiting TB DILI in females with an overall incidence of 2\% [37]. Mahmood et al. [20] reported a higher frequency of TB DILI in females than males (26.3\% vs. 19.7\%). In contrast, this study along with other recent studies [38]-[40] did not show female gender to be a threat for developing TB DILI. In this study, diabetes was established to be a risk factor for TB DILI. Srivastava RK, et al. [41] also reported that diabetes amplifies TB DILI likewise our study. Earlier studies [42] [43] on the subject also depicted diabetes as a risk factor for hepatotoxicity. An earlier study [22] defined our study and did not prove diabetes as a risk factor. Malnutrition is considered to be a risk factor for TB DILI since long. Current reports keep on validating the significance of low albumin to be a risk factor as well as an indicator of malnutrition mutually for TB DILI. In this study various degree of malnutrition were assessed by SGA scheme and patients were categorized into stage A, B and C. Malnourished patients with SGA B (Odd ratio $=0.29,95 \%$ CI $0.19-0.65 \mathrm{p}=0.001$ ) and SGA C (odd ratio $=6.69,95 \% \mathrm{CI}, 3.63-14.41, \mathrm{p}=0.001$ ) category were at greater risk of TB DILI in this study. The SGA technique uses the clinical information collected during history taking and physical examination to determine nutritional status without resorting to objective measurements like anthropometry [19] [44]. SGA method of assessment has been used successfully to assess nutritional status, in general medical and surgical patients. Earlier studies demonstrated three-fold elevated risk of increasing TB DILI in patients with hypoalbuminemia $(<3.5 \mathrm{mg} / \mathrm{dl})$ [5] [31]. Recently weight loss has been implicated as a significant risk factor for DILI [45].

Concomitant use of hepatotoxic drugs with ATT were also instituted to be a significant risk factor for DILI 
$($ Odd ratio $=5.34$, CI 95\%, $2.69-10.68, \mathrm{p}=0.000)$. Previous studies [22] [46] also proved parallel hepatotoxic drugs as a risk factor for TB DILI. In current study, extrapulmonary TB was found to be a risk factor for TB DILI. An earlier study also showed a superior risk of TB DILI in extrapulmonary TB when compared to pulmonary TB [47]. A recent Indian study has also shown that extrapulmonary TB has more ATT DILI compared to pulmonary TB [48]. Concomitant hepatotoxic drugs and severe malnutrition were found to be independently associated with TB DILI when assessed by multivariate analysis. Earlier studies [22] [25] [48] were also congruent with this study for independent risk factors but malnutrition was assessed by low serum albumin rather than SGA staging. Among the risk factors for TB DILI, oxidative stress parameters like Malondialdehyde (MDA), Glutathione (GSH) and Superoxide dismutase (SOD) are also important. The oxidative stress parameters were not determined here, main shortcoming in this study as their serum level also should have been determined before and during the treatment. This would have helped in further understanding of endogenous factors for TB DILI.

\section{Conclusion}

TB DILI is a frequently reported medical problem in patients with various types of tuberculosis. All patterns of DILI were present where hepatocellular and mixed were elaborated the commonest. TB DILI ranges from mild injury to very severe or fatal liver damage. Age more than 35 years, malnutrition, extra pulmonary TB and concomitant use of hepatotoxic drugs were identified risk factors for TB DILI. Severe degree of malnutrition and concomitant use of hepatotoxic drugs were found to be an independent risk factor for TB DILI.

\section{Conflict of Interest}

Authors have no conflict of interest.

\section{References}

[1] World Health Organization (2010) Global Tuberculosis Control: WHO Report 2010. WHO Press, Geneva, 5 p.

[2] World Health Organization (2012) Global Tuberculosis Control: WHO Report 2012. http://apps.who.int/iris/bitstream/10665/75938/1/9789241564502_eng.pdf

[3] Corbett, E.L., Watt, C.J., Walker, N., et al. (2003) The Growing Burden of Tuberculosis: Global Trends and Interactions with the HIV Epidemic. Archives of Internal Medicine, 163, 1009-1021. http://dx.doi.org/10.1001/archinte.163.9.1009

[4] Huang, Y.-S., Chern, H.-D., Su, W.-J., et al. (2002) Polymorphism of the N-Acetyltransferase 2 Gene as a Susceptibility Risk Factor for Antituberculosis Drug-Induced Hepatitis. Hepatology, 35, 883-889. http://dx.doi.org/10.1053/jhep.2002.32102

[5] Sharma, S.K., Balamurugan, A., Saha, P.K., Pandey, R.M. and Mehra, M.K. (2002) Evaluation of Clinical and Immunogenetic Risk Factors for the Development of Hepatotoxicity during Antituberculosis Treatment. American Journal of Respiratory and Critical Care Medicine, 166, 916-919. http://dx.doi.org/10.1164/rccm.2108091

[6] Marrary, W.C. (2012) Alcohol and Drug Induced Liver Injury. In: Shiff, E.R., Maddrey, W.C., Sorell, M.F., Eds., Schiff's Diseases of Liver, John Wiley \& Sons Ltd., 653-784.

[7] Saukkonen, J.J., Cohn, D.L., Jasmer, R.M., et al. (2006) An Official ATS Statement: Hepatotoxicity of Antituberculosis Therapy. American Journal of Respiratory and Critical Care Medicine, 174, 935-952. http://dx.doi.org/10.1164/rccm.200510-1666ST

[8] Tostmann, A., Boeree, M.J., Aarnoutse, R.E., de Lange, W.C., van der Ven, A.J. and Dekhuijzen, R. (2008) Antituberculosis Drug-Induced Hepatotoxicity: Concise Up-to-Date Review. Journal of Gastroenterology, 23, $192-202$. http://dx.doi.org/10.1111/j.1440-1746.2007.05207.x

[9] Baghaei, P., Tabarsi, P., Chitsaz, E., Saleh, M., et al. (2010) Incidence, Clinical and Epidemiological Risk Factors, and Outcome of Drug-Induced Hepatitis Due to Antituberculous Agents in New Tuberculosis Cases. American Journal of Therapeutics, 17, 17-22. http://dx.doi.org/10.1097/MJT.0b013e31818f9eae

[10] Chitturi, S. and Farrell, G. (2002) Drug-Induced Liver Disease. In: Schiff, E.R., Sorrell, M.F., Maddrey, W.C., Eds., Schiff's Diseases of the Liver, Lippincott, Williams \&Wilkins, 1059-1128.

[11] Larrey, D. (2002) Epidemiology and Individual Susceptibility to Adverse Drug Reactions Affecting the Liver. Seminars in Liver Disease, 22, 145-155. http://dx.doi.org/10.1055/s-2002-30101

[12] Senousy, B.E., Belal, S.I. and Draganov, P.V. (2010) Hepatotoxic Effects of Therapies for Tuberculosis. Nature Re- 
views Gastroenterology \& Hepatology, 7, 543-556. http://dx.doi.org/10.1038/nrgastro.2010.134

[13] Kumar, R., Shalimar, B.V., Khanal, S., Sreenivas, V., Gupta, S.D., et al. (2010) Antituberculosis Therapy-Induced Acute Liver Failure: Magnitude, Profile, Prognosis, and Predictors of Outcome. Hepatology, 51, 1665-1674. http://dx.doi.org/10.1002/hep.23534

[14] Kaona, F.A., Tuba, M., Siziya, S. and Sikaona, L. (2004) An Assessment of Factors Contributing to Treatment Adherence and Knowledge of TB Transmission among Patients on TB Treatment. BMC Public Health, 4, 68. http://dx.doi.org/10.1186/1471-2458-4-68

[15] Hussain, Z., Kar, P. and Hussain, S.A. (2003) Antituberculosis Drug-Induced Hepatitis: Risk Factors, Prevention and Management. Indian Journal of Experimental Biology, 41, 1226-1232.

[16] Breen, R.A., Miller, R.F., Gorsuch, T., et al. (2006) Adverse Events and Treatment Interruption in Tuberculosis Patients with and without HIV Co-Infection. Thorax, 61, 791-794.

[17] National Guidelines for Control of Tuberculosis in Pakistan, January 2015. http://ntp.gov.pk/uploads/NATIONAL_GUIDELINE_ON_TB_CASE_MANAGEMENT_REV_JAN_2015.pdf

[18] Aithal, G.P., Watkins, P.B., Andrade, R.J., et al. (2011) Case Definition and Phenotype Standardization in DrugInduced Liver Injury. Clinical Pharmacology \& Therapeutics, 89, 806-815. http://dx.doi.org/10.1038/clpt.2011.58

[19] Detsky, A.S., McLaughlin, J.R., Baker, J.P., et al. (1987) What Is Subjective Global Assessment of Nutritional Status? Journal of Parenteral and Enteral Nutrition, 11, 8-13. http://dx.doi.org/10.1177/014860718701100108

[20] Mahmood, K., Hussain, A., Jairamani, K.L. and Talib, A. (2007) Hepatotoxicity with Antituberculosis Drugs: The Risk Factors. Pakistan Journal of Medical Sciences, 23, 33-38.

[21] Hno, M., Yamaguchi, I., Yamamoto, I., et al. (2000) Slow N-Acetyltransferase 2 Genotype Affects the Incidence of INH and RMP-Induced Hepatotoxicity. International Journal of Tuberculosis and Lung Disease, 4, 256-261.

[22] Khalili, H., Dashti-Khavidaki, S., Rasoolinejad, M., et al. (2009) Anti-Tuberculosis Drugs Related Hepatotoxicity; Incidence, Risk Factors, Pattern of Changes in Liver Enzymes and Outcome. DARU, 17, 163-167.

[23] Steele, M.A., Burk, R.F. and Des Prez, R.M. (1991) Hepatitis with INH and RMP: A Meta-Analysis. Chest, 99, 465471. http://dx.doi.org/10.1378/chest.99.2.465

[24] Makhlouf, H.A., Helmy, A., Fawzy, E., El-Attar, M. and Rashed, H.A. (2008) A Prospective Study of Antituberculous Drug-Induced Hepatotoxicity in an Area Endemic for Liver Diseases. Hepatology International, 2, 353-360. http://dx.doi.org/10.1007/s12072-008-9085-y

[25] Shang, P., Xia, Y., Liu, F., Wang, X., et al. (2011) Incidence, Clinical Features and Impact on Anti-Tuberculosis Treatment of Anti-Tuberculosis Drug Induced Liver Injury (ATLI) in China. PLoS ONE, 6, e21836. http://dx.doi.org/10.1371/journal.pone.0021836

[26] Shaikh, M.A., Yakta, D.E. and Shaikh, D. (2012) Frequency of Hepatotoxicity during Anti-Tuberculous Treatment at Medical Unit of Lumhs Sindh. Medical Channel, 18, 20-23.

[27] Wagan, M.A., Shah, S., Khokar, G.N. and Memon, A.R. (2008) A Study on Hepatic Dysfunction during Dots Therapy for Pulmonary Tuberculosis. Pakistan Journal of Pharmacology, 25, 7-12.

[28] Sharma, S.K., Singala, R., Sadra, P., et al. (2010) Safety of 3 Different Reintroduction Regimens of Antituberculosis Drugs after Development of Antituberculosis Treatment-Induced Hepatotoxicity. Clinical Infectious Diseases, 50, 833839. http://dx.doi.org/10.1086/650576

[29] Tahaoðlu, K., Ataç, G., Sevim, T., et al. (2001) The Management of Anti-Tuberculosis Drug-Induced Hepatotoxicity. International Journal of Tuberculosis and Lung Disease, 5, 65-69.

[30] Saukkonen, J. (2010) Challenges in Reintroducing Tuberculosis Medications after Hepatotoxicity. Clinical Infectious Diseases, 50, 840-842. http://dx.doi.org/10.1086/650577

[31] Singla, R., Sharma, S.K., Mohan, A., et al. (2010) Evaluation of Risk Factors for Antituberculosis Treatment Induced Hepatotoxicity. Indian Journal of Medical Research, 132, 81-86.

[32] Smith, C.A., Wadelius, M., Gough, A.C., Harrison, D.J., Wolf, C.R. and Rane, A. (1997) A Simplified Assay for the Arylamine $N$-Acetyltransferase 2 Polymorphism Validated by Phenotyping with Isoniazid. Journal of Medical Genetics, 34, 758-760. http://dx.doi.org/10.1136/jmg.34.9.758

[33] Dufour, D.R., Lott, J.A., Nolte, F.S., Gretch, D.R., Koff, R.S. and Seeff, L.B. (2000) Diagnosis and Monitoring of Hepatic Injury: I. Performance Characteristics of Laboratory Tests. Clinical Chemistry, 46, 2027-2049.

[34] Steele, M.A., Burk, R.F. and Des Prez, R.M. (1991) Toxic Hepatitis with Isoniazid and Rifampin. A Meta-Analysis. Chest, 99, 465-471. http://dx.doi.org/10.1378/chest.99.2.465

[35] Shakya, R., Rao, B.S. and Shrestha, B. (2004) Evaluation of Risk Factors for Anti-Tuberculosis Drug Induced Hepatotoxicity in Nepalese Population. Annals of Pharmacotherapy, 38, 1074-1079. http://dx.doi.org/10.1345/aph.1D525 
[36] Younossian, A.B., Rochat, T., Ketterer, J.P., Wacker, J. and Janssens, J.P. (2005) High Hepatotoxicity of Pyrazinamide and Ethambutol for Treatment of Latent Tuberculosis. European Respiratory Journal, 26, 462-464. http://dx.doi.org/10.1183/09031936.05.00006205

[37] Papastavros, T., Dolovich, L.R., Holbrook, A., Whitehead, L. and Loeb, M. (2002) Adverse Events Associated with Pyrazinamide and Levofloxacin in the Treatment of Latent Multidrug-Resistant Tuberculosis. Canadian Medical Association Journal, 167, 131-136.

[38] Devarbhavi, H., Dierkhising, R. and Kremers, W.K. (2010) Antituberculosis Therapy Drug-Induced Liver Injury and Acute Liver Failure. Hepatology, 52, 798-799. http://dx.doi.org/10.1002/hep.23805

[39] Devarbhavi, H., Dierkhising, R., Kremers, W.K., Sandeep, M.S., Karanth, D. and Adarsh, C.K. (2010) Single-Center Experience with Drug-Induced Liver Injury from India: Causes, Outcome, Prognosis, and Predictors of Mortality. American Journal of Gastroenterology, 105, Article ID: 2396404. http://dx.doi.org/10.1038/ajg.2010.287

[40] Fountain, F.F., Tolley, E., Chrisman, C.R. and Self, T.H. (2005) Isoniazid Hepatotoxicity Associated with Treatment of Latent Tuberculosis Infection: A 7-Year Evaluation from a Public Health Tuberculosis Clinic. Chest, 128, 116-123. http://dx.doi.org/10.1378/chest.128.1.116

[41] Srivastava, R.K., Sharma, S., Verma, S., Arora, B. and Lal, H. (2008) Influence of Diabetes on Liver Injury Induced by Antitubercular Drugs and on Silymarin Hepatoprotection in Rats. Methods and Findings in Experimental and Clinical Pharmacology, 30, 731-737. http://dx.doi.org/10.1358/mf.2008.30.10.1316824

[42] De Leve, L. and Kaplowitz, N. (2000) Prevention and Therapy of Drug-Induced Hepatic Injury. In: Wolfe, M., Ed., Therapy of Digestive Disorders, WB Saunders, Philadelphia, 334-348.

[43] Zimmerman, H. (1999) Drug-Induced Liver Disease. In: Schiff, E., Sorell, M. and Madding, W., Eds., Schiff's Diseases of the Liver, Lippincott-Raven Publishers, Philadelphia, 973-1064.

[44] Makhija, S. and Baker, J. (2008) The Subjective Global Assessment: A Review of Its Use in Clinical Practice. Nutrition in Clinical Practice, 23, 405-409. http://dx.doi.org/10.1177/0884533608321214

[45] Warmelink, I., ten Hacken, N.H., van der Werf, T.S. and van Altena, R. (2011) Weight Loss during Tuberculosis Treatment Is an Important Risk Factor for Drug-Induced Hepatotoxicity. British Journal of Nutrition, 105, 400-408. http://dx.doi.org/10.1017/S0007114510003636

[46] Yew, W.W. and Leung, C.C. (2006) Anti-Tuberculosis Drugs and Hepatotoxicity. Respirology, 11, 699-707. http://dx.doi.org/10.1111/j.1440-1843.2006.00941.x

[47] Marzuki, O.A., Fauzi, A.R.M., Ayoubm, S. and Kamarul Imran, M. (2008) Prevalence and Risk Factors of Antituberculosis Drug-Induced Hepatitis in Malaysia. Singapore Medical Journal, 49, 688-693.

[48] Ambreen, K., Sharma, R., Singh, K.P., Khan, F.H. and Kumar, S. (2012) Risk Factors for Antituberculosis DrugInduced Hepatotoxicity and Its Association with Oxidative Stress in North Indian Population. Annals of Tropical Medicine and Public Health, 5, 574-580. http://dx.doi.org/10.4103/1755-6783.109278 